\title{
Mucopolysaccharidosis Type IVA
}

\section{N-Acetylgalactosamine-6-Sulfate Sulfatase Exonic Point Mutations in Classical Morquio and Mild Cases}

\author{
Seiji Fukuda, * Shunji Tomatsu, * Michiya Masue, “ Kazuko Sukegawa, * Hideki Iwata, * Tatsuya Ogawa, * \\ Yoshihiro Nakashima, * Toshinori Hori, * Atsushi Yamagishi, * Yuko Hanyu, * \\ Keiichi Morooka, ${ }^{*}$ Tadashi Kiman, "Takashi Hashimoto," and Tadao Orii* \\ ${ }^{*}$ Department of Pediatrics, Gifu University School of Medicine, 40 Tsukasa-machi, Gifu 500, Japan; ${ }^{\ddagger}$ First Department of Pediatrics, \\ Toho University School of Medicine, Tokyo 153, Japan; ${ }^{\S}$ Koriyama Ryoikuen, Koriyama 963, Japan; and "Department of Biochemistry, \\ Shinshu University School of Medicine, Matsumoto, Nagano 390, Japan
}

\begin{abstract}
Mucopolysaccharidosis type IVA (MPS IVA) results from a genetic deficiency of $N$-acetylgalactosamine-6-sulfate (GalNAc6S) sulfatase. We have identified two different exonic mutations causing GalNAc6S sulfatase deficiency in two unrelated Japanese families, in one patient with classical Morquio disease, and in two brothers with a mild form of MPS IVA. The nucleotide sequence of the full-length cDNA derived from a patient with classical Morquio disease revealed a two-base deletion at nucleotide position 1343-1344 (1344-1345 or 13451346 ) that altered the reading frame (designated 1342delCA). This mutation, inherited from the proband's consanguineous parents, was revealed by TaqI restriction analysis of a cDNA fragment amplified by the polymerase chain reaction. In the proband with the mild form of the disease, a $\mathbf{C}$ to $\mathbf{G}$ transversion at nucleotide 667 predicted the substitution of Lys for Asn ${ }^{204}$ (N204K). Since a new AluI site was created by the N204K mutation, restriction analysis indicated that the affected brothers were homozygous for this mutation, as confirmed by the finding that both their parents had this lesion. Transient expression in GalNAc6S sulfatase deficient fibroblasts of these two mutant alleles showed completely deficient or markedly decreased enzyme activities, thereby indicating that these two mutations were responsible for the enzyme deficiency. ( J. Clin. Invest. 1992. 90:1049-1053.) Key words: cDNA cloning $\bullet$ mutation • modified PCR • expression • clinical and genetic heterogeneity
\end{abstract}

\section{Introduction}

Mucopolysaccharidosis type IVA (MPS IVA) ${ }^{1}$ is an autosomal recessive lysosomal storage disorder caused by a deficiency of $\mathrm{N}$-acetylgalactosamine-6-sulfate (GalNAc6S) sulfatase (E.C.3.1.6.4), also known as galactose-6-sulfate sulfatase

Address reprint requests to Dr. S. Fukuda, Department of Pediatrics, Gifu University School of Medicine, Gifu, Gifu 500, Japan.

Received for publication 17 January 1992 and in revised form 4 April 1992.

1. Abbreviations used in this paper: CRM, cross-reacting material; GaINAc6S; $N$-acetylgalactosamine-6-sulfate; MEM, minimal essential medium; MPS IVA, mucopolysaccharidosis type IVA; PCR, polymerase chain reaction.

J. Clin. Invest.

(C) The American Society for Clinical Investigation, Inc.

$0021-9738 / 92 / 09 / 1049 / 05 \$ 2.00$

Volume 90, September 1992, 1049-1053
(1-3). GalNAc6S sulfatase is a lysosomal enzyme which hydrolyzes the sulfate ester group of GalNAc6S at the nonreducing end of chondroitin-6-sulfate, and that of galactose-6-sulfate at the nonreducing end of keratan sulfate (4). A defect in this enzyme leads to accumulation of undegraded glycosaminoglycans in lysosomes. Clinically, MPS IVA can be divided into several subtypes: besides the severe (classical) form (so-called Morquio disease) (5), there also exists an intermediate (6) and a mild form (7-10). Classical Morquio disease is a prototype of chondroosteodystrophy (spondyloepiphyseal dysplasia), characterized by specific spondyloepiphyseal dysplasia, short trunk dwarfism, coxa valga, odontoid hypoplasia, corneal opacities, preservation of intelligence, and excessive urinary excretion of keratan sulfate and chondroitin-6-sulfate $(5,11)$.

Biochemical studies of the genetic heterogeneity in MPS IVA have been hampered because GalNAc6S sulfatase is difficult to purify and specific antibodies have been difficult to produce. Available enzymatic assays do not differentiate the mild from the severe form. Although the enzymatic diagnosis of affected patients with MPS IVA can be made, enzymatic carrier detection is less reliable because of a marked overlap of GalNAc6S sulfatase activity in fibroblasts or leukocytes from obligate heterozygotes and normal controls. We purified GalNAc6S sulfatase from human placenta and obtained a polyclonal antibody against the enzyme (12). Human GalNAc6S sulfatase is an oligomer with a molecular mass of $120 \mathrm{kD}$ and consists of 40 and $15 \mathrm{kD}$ polypeptides linked by a disulfide bond (12). Some patients with classical MPS IVA have no detectable cross-reacting material (CRM) (12), while others have CRM with no detectable enzyme activity (unpublished data). These findings and the heterogeneity of phenotypes suggest different mutations, each of which may have different effects on the active enzyme.

Investigations into the molecular basis of the genetic heterogeneity in MPS IVA have been facilitated by the isolation and characterization of the full-length cDNA encoding human GalNAc6S sulfatase (13). The 2339-bp cDNA contains an open reading frame of $1566 \mathrm{bp}$, which encodes 522 amino acids, including a signal peptide of 26 residues.

In this communication, the first two exonic mutations in the GalNAc6S sulfatase gene causing severe and milder forms of MPS IVA are described. These mutations were confirmed in cDNA and carrier detection can now be made using a simple and rapid polymerase chain reaction (PCR)-based assay.

\section{Methods}

Patients and cell lines. Three Japanese patients from two unrelated families were studied. Case 1 was a 31 -yr-old woman who had classical Morquio disease. Her parents were first cousins and her clinical fea- 
tures were typical for Morquio disease, with spondyloepiphyseal dysplasia, short stature, normal intelligence, and excretion of keratan sulfate and chondroitin-6-sulfate in the urine. The GalNAc6S sulfatase activity in her fibroblasts was severely deficient, and CRM was not detected. Cases 2 and 3, patients aged 25 and 29, respectively, were also the offsprings of first cousin parents. Clinical manifestations of these patients have been described elsewhere $(7,14-16)$. They were cases of late-onset disease that were mild compared to classically affected patients. The residual activities in their fibroblasts were below $2 \%$ of the normal, and only the $60-\mathrm{kD}$ precursor polypeptide was detected by polyclonal antibodies against the purified enzyme. Fibroblasts derived from patients, family members, and a normal control were cultured in Eagle's minimum essential medium (MEM) containing 10\% FCS.

Materials. Restriction endonucleases, T4 polymerase, T4 ligase, T4 kinase, and Taq polymerase were purchased from Takara Shuzo (Kyoto, Japan) and Murine leukemia virus reverse transcriptase was from Bethesda Research Laboratories (Gaithersburg, MD). [ $\left.\alpha^{-32} \mathrm{P}\right]-$ dCTP and $\alpha^{-3}$ S-dATP were obtained from Amersham (Buckinghamshire, UK). PCR primers and specific oligonucleotide primers were synthesized with an automated DNA synthesizer (model 380A; Applied Biosystems, Inc., Foster City, CA).

$R N A$ preparation and Northern blot analysis. Total RNA was extracted from fibroblasts or peripheral leukocytes using the guanidinium isothiocyanate procedure (17). Approximately $80 \mu \mathrm{g}$ of total RNA derived from patients and normal controls was electrophoresed using the glyoxal method (18), transferred onto nylon membrane filters and hybridized with full-length cDNA for GalNAc6S sulfatase labeled with $\left[\alpha{ }^{32} \mathrm{P}\right] \mathrm{dCTP}(18)$.

Amplification of cDNA by PCR, subcloning, and sequencing. $5 \mu \mathrm{g}$ of total RNA were reverse-transcribed with Murine leukemia virus reverse transcriptase and GalNAc6S sulfatase cDNA specific primers or random hexanucleotide primers, according to manufacturer's instructions. Half of the cDNA product was annealed to $50 \mathrm{pmol}$ each of the sense and antisense primers, and 40 cycles of the PCR were performed. Each cycle consisted of 1 min denaturation at $94^{\circ} \mathrm{C}, 2$ min annealing at $54^{\circ} \mathrm{C}$, and $3 \mathrm{~min}$ extension at $72^{\circ} \mathrm{C}$. The final extension was for $7 \mathrm{~min}$ (Fig. 1) (19). The PCR products were subcloned into pUC13, and
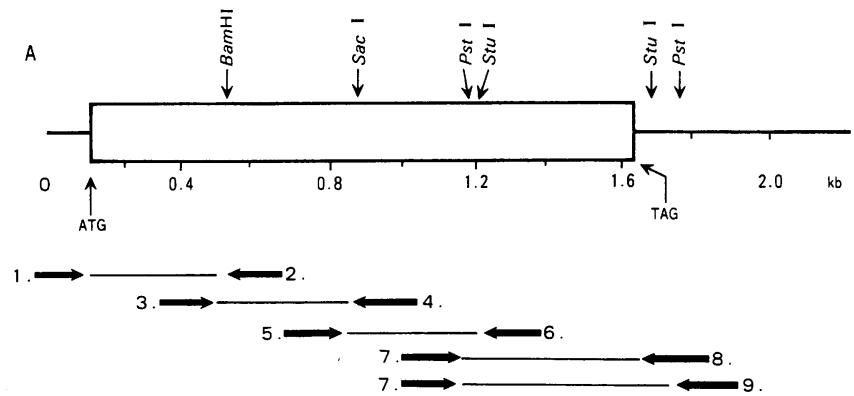

B

\begin{tabular}{ccc} 
Primer & \multicolumn{1}{c}{ Sequence } & Position \\
\hline 1. & 5' -ATGAATTCGGCTCCCGTGGTTGCC-3' & $(39-55)$ \\
2. & 5' -CATAAGGTCCAAAGTGGCAGT-3' & $(566-546)$ \\
3. & 5' - TGAAGCACGGATTTGATGAGT-3' & $(510-530)$ \\
4. & 5' - TGTCCGACGTGAAGAAGACGA-3' & $(920-900)$ \\
5. & 5' - AGATTGATGACAGCATTGGGA-3' & $(834-854)$ \\
6. & 5' - TTCCAGATTGTGAGTTGTGAC-3' & $(1354-1334)$ \\
7. & 5' -CATTGATGGCCTCAACCTCCT-3' & $(1141-1161)$ \\
8. & 5' -GCCAACTGGAGATTCTAGGC-3' & $(1666-1647)$ \\
9. 5' -GGCAGGTGGAATTGTGCAGTC-3' & $(1777-1757)$
\end{tabular}

Figure 1. Strategy for PCR amplification. Nucleotide numbers are indicated as described previously (13). $(A)$ The cDNA fragments were synthesized with four pairs of primers. Restriction map for GalNAc6S sulfatase cDNA and the relative positions and direction of extension for the primers are indicated. $(B)$ Sequence of oligonucleotide primers. Primer 1 contains an EcoRI linker sequence at the 5' end. several independent clones were sequenced in both orientations, using the dideoxy chain termination method (20).

Transient expression in enzyme-deficient fibroblasts. Specific cDNA fragments in $\mathrm{pMF}(\mathrm{N})$, the expression vector pCAGGS coupled
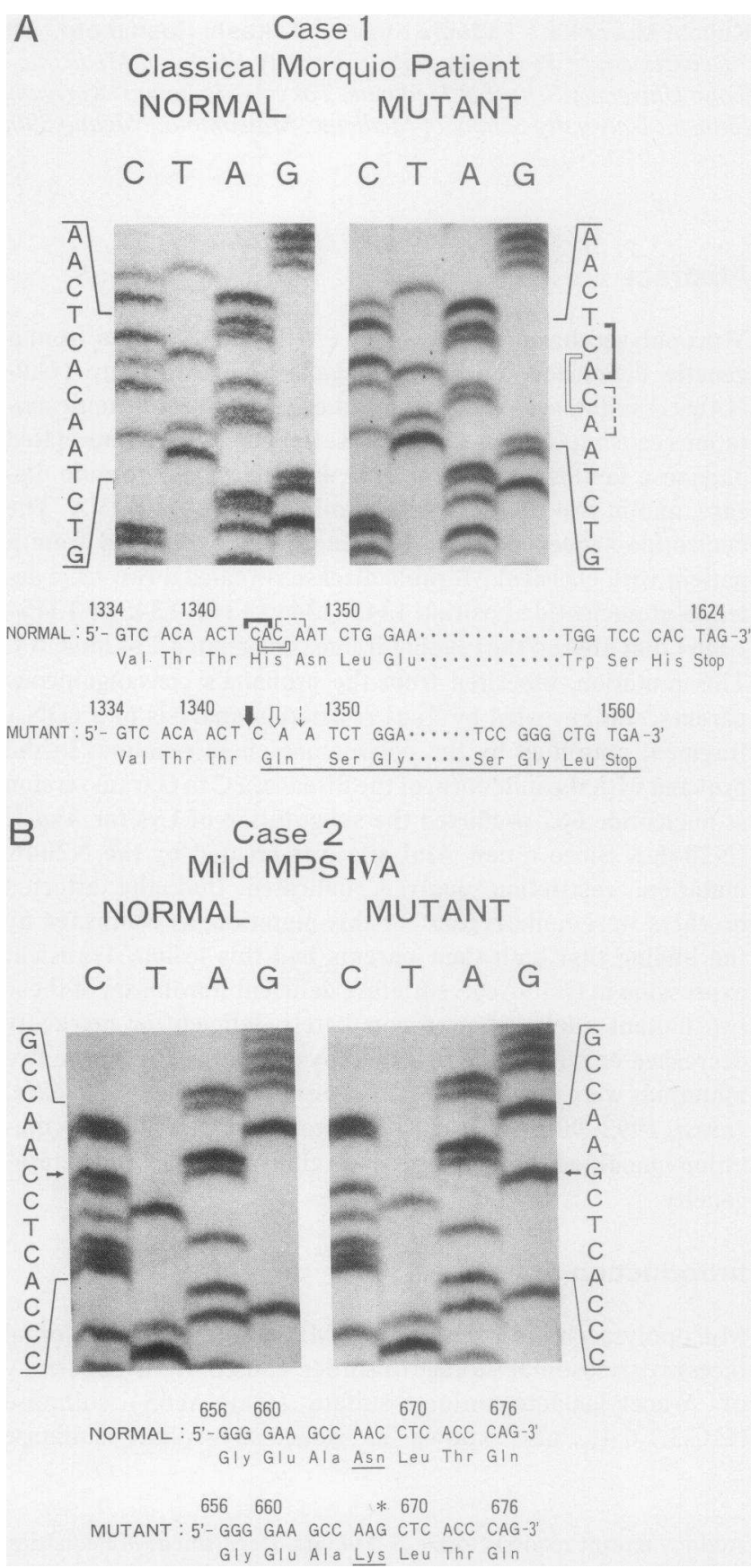

Figure 2. Nucleotide sequence including the mutation sites of normal and mutated cDNA. $(A)$ The two-base deletion that altered the reading frame in classical Morquio patient (1342delCA, case 1). Three possible deletion sites are bracketed $(\square$, nt 1343-1344; $\square$, nt 1344 $1345 ; \Gamma^{---1}$, nt 1345-1346). Arrows indicate the corresponding positions in the mutant allele. The deduced amino acid sequence as a result of frame shift are underlined. $(B)$ The $C$ to $G$ transversion that resulted in the replacement of Asn ${ }^{204}$ by Lys in mild type patient (N204K, case 2) and corresponding amino acid alteration. The arrows and asterisks indicate the mutation site and the substituted $\mathrm{G}$ residue in the mutant sequence, respectively. The altered amino acids are underlined. 


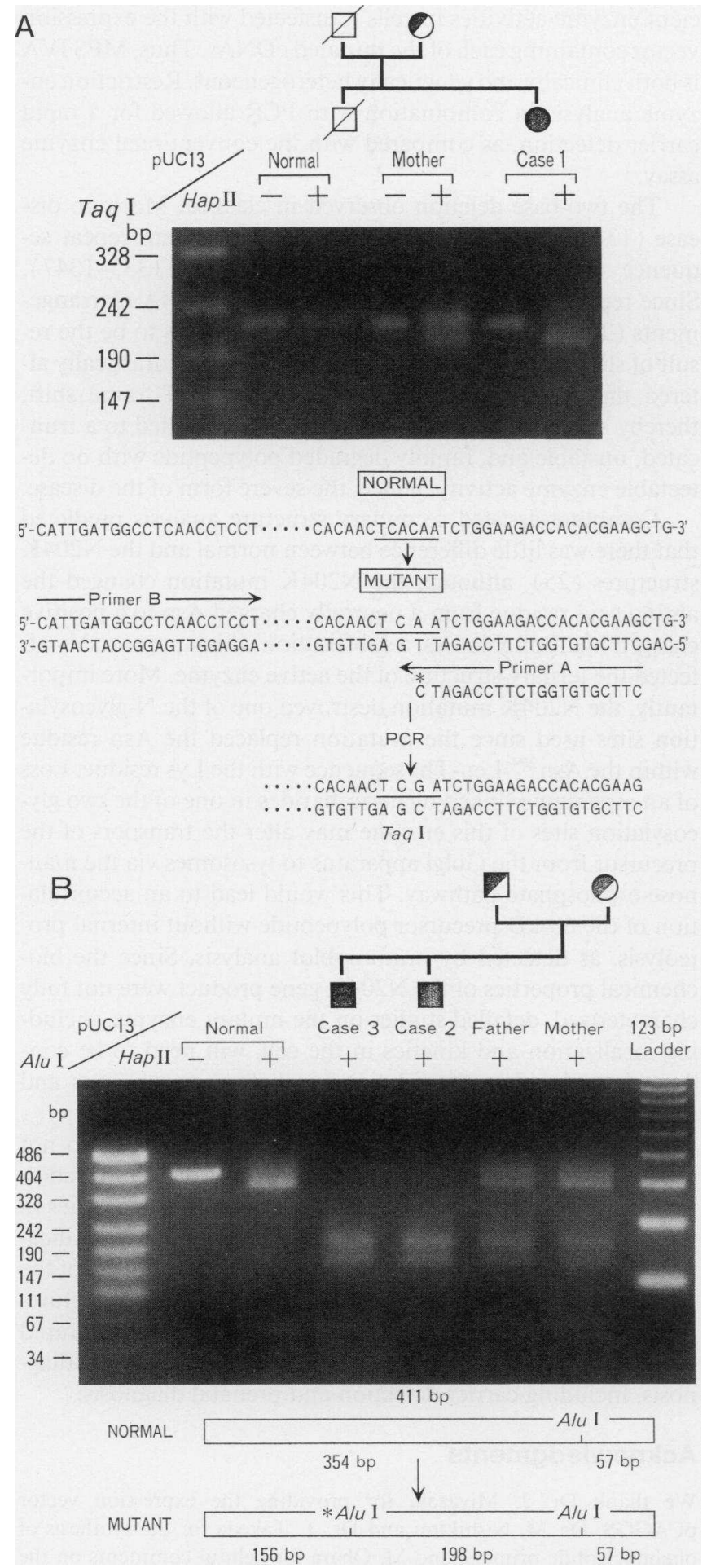

Figure 3. Family studies done using PCR and restriction enzyme analysis. $(A)$ Upper panel shows the TaqI digestion of the cDNA amplified using a modified PCR method. Fragments were obtained from the classical Morquio patient (case 1), her mother, and a normal control. The resulting PCR products both before $(-)$ and after $(+)$ digestion were electrophoresed and visualized in a $4 \%$ NuSieve agarose gel with ethidium bromide staining. The normal cDNA fragment was not digested with TaqI, while the mutated cDNA fragment was completely digested to 203 and $21 \mathrm{bp}$ fragments. The 21 -bp fragments are not seen in this gel.

Lower panel shows the strategy for introducing TaqI restriction site on the mutant allele. Partial nucleotide sequence including the with the normal cDNA, were replaced with the corresponding fragment carrying each of the identified mutations. The plasmids were designated pMF (1342delCA), including a two-base deletion that was found in the patient case 1 sequence, and $\mathrm{pMF}(\mathrm{N} 204 \mathrm{~K})$, harboring the Lys for $\mathrm{Asn}^{204}$ substitution observed in patient cases 2 and 3, respectively. Using a commercial reagent using liposomes (Wako, Osaka, Japan ), each vector was transfected into GalNAc6S sulfatase-deficient fibroblasts obtained from an unrelated patient with MPS IVA (21). Ga1NAc6S sulfatase activities in the cell extracts were measured $120 \mathrm{~h}$ after transfection, as described in the literature $(12,13)$.

\section{Results}

Identification of mutations in the GalNAc6S sulfatase cDNA. Since the mRNA was apparently of a normal size and abundancy in all subjects as deduced by Northern blot analysis (data not shown), the possibility of promoter and mRNA processing mutations was excluded. Thus, alterations causing the enzyme defect were caused by point mutations, small deletions, or insertions in the coding region. Subsequent sequence analysis indicated different mutations in each patient. In the patient with classical Morquio disease (case 1), a two-base deletion of nucleotides 1343-1344 (1344-1345 or 1345-1346) was observed. This introduced a frameshift in the 3 '-flanking sequence with the occurrence of a termination codon at nucleotides 1558-1560 (designated 1342delCA, Fig. $2 \mathrm{~A}$ ). On the other hand, a $C$ to $\mathrm{G}$ transversion at nucleotide 667 was identified in the sequence from case 2 with a mild form of the disease, the result being the substitution of Lys for Asn at residue 204 (N204K, Fig. 2 B). Other additional mutations, a $\mathrm{T}$ to $\mathrm{C}$ transition at position 763 (case 2), and an $A$ to $G$ transition at position 1486 (cases 1 and 2) were also identified, respectively, but these were silent third base substitutions that were considered to be neutral polymorphisms. No other substitutions were evident that would alter the amino acid sequence. To rule out a Taq-polymerase error, more than five independent clones were sequenced and a new set of experiments was carried out, starting from cDNA synthesis of total RNA.

Characterization of mutations in family members. In case 1 , although neither the normal nor the mutant sequence surrounding the 1342delCA deletion site contained an appropriate restriction site, modification of the PCR method facilitated segregation of the normal and mutant alleles. We prepared a mismatched PCR primer to introduce the TaqI restriction site into the mutant sequence. The mutant allele encompassing the 1141 to 1366 nucleotide sequence was amplified with primer $A$ containing a mismatched $C$ residue at the 3 ' end, and with another primer B, thereby generating a new TaqI site at the deletion site. In contrast, if the normal allele was amplified, the TaqI recognition site did not occur. As shown in Fig. $3 \mathrm{~A}$, the fragment derived from the patient was

deletion site are underlined. The asterisks indicates the mismatched $C$ residue in the downstream primer. $(B)$ AluI digestion of amplified cDNA from two brothers with mild form of MPS IVA (cases 2 and 3) and their parents. The cDNA fragment at bp 510-920 derived from the affected two brothers, their parents, and normal control with (+) or without (-) AluI digestion were electrophoresed in a $2 \%$ agarose gel followed by ethidium bromide staining. The presence of a 354-bp fragment indicates the normal allele, while a 198- and 156-bp fragment indicates the mutant allele. The asterisks represents the AluI site created by the mutation. 
completely digested with TaqI, but the control fragment remained intact and partial digestion was observed in the cDNA derived from the proband's mother. The father and brother were deceased.

The N204K mutation that was found in case 2 produced a new AluI restriction site (ACT $\rightarrow$ A $\underline{G}$ T) in the mutant sequence, and thus could be detected by PCR and restriction enzyme digestion. To examine the inheritance of the N204K mutation and to exclude a cloning artifact, the PCR product including nucleotide 510-920 from the two affected brothers and their parents was amplified and subjected to AluI restriction assay. As shown in Fig. $3 B$, both patients had only the mutant allele, while their parents had both normal and mutant alleles. Thus, cases 1 and 2 were homozygous for their respective mutant alleles and their parents were heterozygous for both normal and mutant alleles, a finding consistent with the inheritance of MPS IVA as an autosomal ressesive trait.

Expression of mutant protein in enzyme-deficient fibroblasts. In a transient expression assay, the enzyme-deficient fibroblasts transfected with pMF $(N)$ had almost the same activity as the normal control fibroblasts. On the other hand, fibroblasts transfected with pMF(1342delCA) had no detectable enzyme activity, while cells with pMF(N204K) expressed $6 \%$ of the activity of the fibroblasts transfected with pMF(N) (Table I).

\section{Discussion}

Our results provide evidence that two different mutations (1342delCA and N204K) were responsible for the deficient $\mathrm{N}$-acetylgalactosamine-6-sulfate sulfatase activity causing two different phenotypes of MPS IVA. These mutations were confirmed by the demonstration that these alleles encoded defi-

Table I. Activity of GalNAc6S Sulfatase in Transfected Enzyme-deficient Fibroblasts

\begin{tabular}{lc}
\hline \multicolumn{1}{c}{ Plasmid } & $\begin{array}{c}\text { GalNAc 6S sulfatase activity } \\
\text { (nmol/mg protein/h) }\end{array}$ \\
\hline Control fibroblast & 18.8 \\
Enzyme-deficient fibroblast & $\mathrm{ND}^{*}$ \\
pMF (1342del CA): (case 1) & $\mathrm{ND}^{*}$ \\
pMF (N204K): (cases 2 and 3) & 0.9 \\
pMF (N): (normal) & 14
\end{tabular}

For the transient expression of mutant GalNAc6S sulfatase in enzyme-deficient fibroblasts, specific fragments in the expression vector pMF $(\mathrm{N})$, derived from pCAGGS coupled with normal cDNA were replaced with a corresponding fragment carrying each of the identified mutations and designated pMF (1342del CA), and pMF (N204K), respectively. Each vector was transfected into the enzyme-deficient fibroblasts obtained from an unrelated patient with MPS IVA (intermediate), as described below. Subconfluent fibroblasts plated $16 \mathrm{~h}$ before transfection were incubated in MEM containing $10 \%$ FCS, and $2 \mathrm{mM}$ liposomes entrapping the plasmid at a concentration of $0.5 \mu \mathrm{g} / \mathrm{ml}$ for $16 \mathrm{~h}$ at $37^{\circ} \mathrm{C}$ in a $5 \% \mathrm{CO}_{2}$ incubator. At the end of transfection, the medium was changed and incubated in MEM for $120 \mathrm{~h}$. GalNAc6S sulfatase activity was assayed with a radiolabeled substrate $(\beta-N$-acetyl-D-galactosamine 6 -sulfate- $(1 \rightarrow 4)-\beta$-D-glucuronic acid- $(1 \rightarrow 3)-N$-acetyl-D- $\left[{ }^{3} \mathrm{H}\right]$ galactosaminitol 6-sulfate) as described $(12,13)$.

* Not detected. cient enzyme activities in cells transfected with the expression vector containing each of the mutated cDNAs. Thus, MPS IVA is both clinically and genetically heterogeneous. Restriction enzyme analysis in combination with PCR allowed for a rapid carrier detection, as compared with the conventional enzyme assay.

The two-base deletion observed in classical Morquio disease (1342delCA) was located in a short tandem repeat sequence of TCACAACTCACAA (nucleotides 1335-1347). Since repeat sequences are often involved in DNA rearrangements (22-24), the 1342delCA deletion appears to be the result of slipped mispairing. The resulting product drastically altered the polypeptide structure, as a result of frame shift, thereby suggesting that the 1342delCA deletion led to a truncated, unstable and, rapidly degraded polypeptide with no detectable enzyme activity, hence, the severe form of the disease.

Computer-assisted secondary structure analysis predicted that there was little difference between normal and the N204K structures (25), although the N204K mutation changed the amino acid residue from a neutrally charged Asn to a positive charged hydrophobic Lys, a substitution which presumably affected the tertiary structure of the active enzyme. More importantly, the N204K mutation destroyed one of the N-glycosylation sites used since the mutation replaced the Asn residue within the Asn ${ }^{204}$-Leu-Thr sequence with the Lys residue. Loss of an asparagine-linked oligosaccharides in one of the two glycosylation sites of this enzyme may alter the transport of the precursor from the Golgi apparatus to lysosomes via the mannose-6-phosphate pathway. This would lead to an accumulation of the $60-\mathrm{kD}$ precursor polypeptide without internal proteolysis, as detected by immunoblot analysis. Since the biochemical properties of the $\mathrm{N} 204 \mathrm{~K}$ gene product were not fully characterized, detailed studies on the mutant enzyme, including localization and kinetics in the cell, will need to be conducted to elucidate the relationship between mutations and enzyme deficiency.

In some patients, residual activities in fibroblasts do not always correlate with clinical severity of the disease. In addition, there is a partial overlap in ranges of enzyme activities in the obligate heterozygote and normal individuals. Identification of mutations causing MPS IVA will allow definition of the genotype/phenotype correlations, as well as the structurefunction relationship of the enzyme. The development of rapid detection methods using PCR will facilitate more precise diagnosis, including carrier detection and prenatal diagnosis.

\section{Acknowledgments}

We thank Dr. J. Miyazaki for providing the expression vector pCAGGS, Dr. M. Nishikimi and Dr. T. Takeda for the synthesis of oligonucleotide primers, and M. Ohara for helpful comments on the manuscript.

This study was supported in part by a Grant-in-Aid for Scientific Research (03265102 and 03557044) from the Ministry of Education, Science, and Culture of Japan; a grant (3A-6) from the National Center of Neurology and Psychiatry of the Ministry of Health and Welfare of Japan; and a grant for Pediatric Research (3-A) from the Ministry of Health and Welfare of Japan.

\section{References}

1. Matalon, R., B. Arbogast, P. Justice, I. K. Brandt, and A. Dorfman. 1974. Morquio's syndrome: deficiency of a chondroitin sulfate $\mathrm{N}$-acetylhexosamine sulfate sulfatase. Biochem. Biophys. Res. Commun. 61:709-715. 
2. Singh, J., N. Di Ferrante, P. Niebes, and D. Tavella. 1976. $\mathrm{N}$-acetylgalactosamine-6-sulfate sulfatase in man: absence of the enzyme in Morquio disease. $J$. Clin. Invest. 57:1036-1040.

3. Horwitz, A. L., and A. Dorfman. 1978. The enzymatic defect in Morquio disease: the specificity of $\mathrm{N}$-acetylhexosamine sulfatases. Biochem. Biophys. Res. Commun. 80:819-825.

4. Di Ferrante, N., L. C. Ginsberg, P. V. Donnelly, and D. T. Di Ferrante. 1978. Deficiencies of glucosamine-6-sulfate or galactose-6-sulfate sulfatase are responsible for different mucopolysaccharidoses. Science (Wash. DC). 199:7981.

5. Morquio, L. 1929. Sur une forme de dystrophie osseuse familiale. Bull. Soc. Pédiat. Paris. 27:145-152.

6. Glössl, J., P. Maroteaux, P. Di Natale, and H. Kresse. 1981. Differen properties of residual $\mathrm{N}$-acetylgalactosamine-6-sulfate sulfatase in fibroblasts from patients with mild and severe forms of Morquio disease type A. Pediatr. Res. 15:976-978.

7. Orii, T., T. Kiman, K. Sukegawa, T. Kanemura, S. Hattori, T. Taga, and K. Ko. 1981. Late onset $\mathrm{N}$-acetylgalactosamine-6-sulfate sulfatase deficiency in two brothers. Connect. Tissue. 13:169-175.

8. Fujimoto, A., and A. L. Horwitz. 1983. Biochemical defect of non-keratansulfate-excreting Morquio syndrome. Am. J. Med. Genet. 15:265-273.

9. Hechit, J. T., C. I. Scott, Jr., T. K. Smith, and J. C. Williams. 1984. Mild manifestations of the Morquio syndrome. Am. J. Med. Genet. 18:369-371.

10. Beck, M., J. Glössl, A. Grubisic, and J. Spranger. 1986. Heterogeneity of Morquio disease. Clin. Genet. 29:325-331.

11. Maroteaux, P., and M. Lamy. 1967. Studying the mucopolysaccharidoses. Lancet. (ii):510.

12. Masue, M., K. Sukegawa, T. Orii, and T. Hashimoto. 1991. N-acetylgalactosamine-6-sulfate sulfatase in human placenta: purification and characteristics. J. Biochem. (Tokyo). 110:965-970.

13. Tomatsu, S., S. Fukuda, M. Masue, K. Sukegawa, T. Fukao, A. Yamagishi, T. Hori, H. Iwata, T. Ogawa, Y. Nakashima, et al. 1991. Morquio disease: isolation, characterization and expression of full-length cDNA for human $\mathrm{N}$-acetylgalactosamine-6-sulfate sulfatase. Biochem. Biophys. Res. Commun. 181:677683.
14. Sukegawa, K., and T. Orii. 1982. Residual activity in fibroblasts from two brothers with the late-onset form of $\mathrm{N}$-acetylgalactosamine-6-sulfate sulfatase deficiency. J. Inherited Metab. Dis. 5:231-232.

15. Orii, T., and T. Taga. 1982. Clinical features and enzymatic diagnosis of Morquio syndrome. Brain Dev. 4:289.

16. Orii, T. 1986. Screening, enzymatic diagnosis and heterozygote identification of genetic mucopolysaccharidoses. Asian Med. J. 29:89-99.

17. Chomczynski, P., and N. Sacchi. 1987. Single-step method of RNA isolation by acid guanidinium thiocyanate-phenol-chloroform extraction. Anal. Biochem. 162:156-159.

18. Sambrook, J., E. F. Fritsch, and T. Maniatis. 1989. Molecular Cloning: A Laboratory Manual. Cold Spring Harbor Laboratory Press, Cold Spring Harbor, NY. 7.39-7.52.

19. Saiki, R. K., S. Scharf, F. Faloona, K. B. Mullis, G. T. Horn, H. A. Erlich, and N. Arnheim. 1985. Enzymatic amplification of $\beta$-globin genomic sequences and restriction site analysis for diagnosis of sickle cell anemia. Science (Wash. DC). 230:1350-1354.

20. Hattori, M., and Y. Sakaki. 1986. Dideoxy sequencing method using denatured plasmid templates. Anal. Biochem. 152:232-238.

21. Koshizaka, T., Y. Hayashi, and K. Yagi. 1989. Novel liposomes for efficient transfection of $\beta$-galactosidase gene into COS-1 cells. J. Clin. Biochem. Nutr. 7:185-192

22. Haraguchi, Y., J. M. Aparicio, R., M. Takiguchi, I. Akaboshi, M. Yoshino, M. Mori, and I. Matsuda. 1990. Molecular basis of arginemia. Identification of two discrete frame-shift deletions in the liver-type arginase gene. J. Clin. Invest. 86:347-350.

23. Gibbs, R. A., P.-N. Nguyen, L. J. McBride, S. M. Koepf, and C. T. Caskey. 1989. Identification of mutations leading to Lesch-Nyhan syndrome by automated direct DNA sequencing of in vitro amplified cDNA. Proc. Natl. Acad. Sci. USA. 86:1919-1923.

24. Canning, S., and T. P. Dryja. 1989. Short, direct repeats at the breakpoints of deletions of the retinoblastoma gene. Proc. Natl. Acad. Sci. USA. 86:50445048 .

25. Chou, P. Y., and G. D. Fasman. 1978. Empirical predictions of protein conformation. Annu. Rev. Biochem. 47:251-276. 